\title{
EXPLICIT SINGULAR VISCOSITY SOLUTIONS OF THE ARONSSON EQUATION
}

\author{
NIKOLAOS I. KATZOURAKIS
}

\begin{abstract}
We establish that when $n \geq 2$ and $H \in C^{1}\left(\mathbb{R}^{n}\right)$ is a Hamiltonian such that some level set contains a line segment, the Aronsson equation $D^{2} u: H_{p}(D u) \otimes H_{p}(D u)=0$ admits explicit entire viscosity solutions. They are superpositions of a linear part plus a Lipschitz continuous singular part which in general is non- $C^{1}$ and nowhere twice differentiable. In particular, we supplement the $C^{1}$ regularity result of Wang and Yu [W-Y] by deducing that strict level convexity is necessary for $C^{1}$ regularity of solutions.

RÉSUmÉ. Nous démontrons que, pour $n \geq 2$ et un Hamiltonien $H \in C^{1}\left(\mathbb{R}^{n}\right)$ tel qu'au moins une de ses lignes de niveau contienne un segment de droite, l'équation de Aronsson $D^{2} u: H_{p}(D u) \otimes H_{p}(D u)=0$ admet des solutions de viscosité explicites définies sur $\mathbb{R}^{n}$. Elles sont superpositions d'une partie linéaire et d'une partie continue, lipschitzienne, singulière qui, en général, n'est pas $C^{1}$ et est nulle part deux fois dérivable. Plus précisément, nous complétons le résultat de régularité établit par Wang et $\mathrm{Yu}[\mathrm{W}-\mathrm{Y}]$ en montrant que la stricte convexité des lignes de niveau est nécessaire pour que les solutions soient $C^{1}$.
\end{abstract}

\section{INTRODUCTION}

Let $H \in C^{1}\left(\mathbb{R}^{n}\right)$ be a Hamiltonian function and $n \geq 2$. We discuss aspects of the $C^{1}$ regularity problem of viscosity solutions to the Aronsson equation, which is defined on smooth $u \in C^{2}\left(\mathbb{R}^{n}\right)$ by

$$
\mathcal{A}[u]:=D^{2} u: H_{p}(D u) \otimes H_{p}(D u)=0 .
$$

Here, $\mathcal{A}[u]$ is understood as $\sum_{i, j=1}^{n} D_{i j}^{2} u H_{p_{i}}(D u) H_{p_{j}}(D u)$ and $H_{p_{i}}=D_{p_{i}} H$. Formula (1) defines a quasilinear highly degenerate elliptic PDE. It arises in $L^{\infty}$ variational problems of the supremal functional $E_{\infty}(u, \Omega):=\|H(D u)\|_{L^{\infty}(\Omega)}$, as well as in other contexts (Barron-Evans-Jensen [BEJ]). When $H(p)=\frac{1}{2}|p|^{2}$, (1) reduces to the $\infty$-Laplacian:

$$
\Delta_{\infty} u:=D^{2} u: D u \otimes D u=0 .
$$

Under reasonable convexity, coercivity and regularity assumptions on $H$, there exists a unique continuous solution of the Dirichlet problem with Lipschitz boundary data, interpreted in the viscosity sense of Crandall-Ishii-Lions [CIL]. Moreover, any continuous viscosity solution to (1) is actually Lipschitz continuous. The $C^{1}$ regularity problem for (1) however remains open. Wang and $\mathrm{Yu}[\mathrm{W}-\mathrm{Y}]$ established that when $n=2, H$ is in $C^{2}\left(\mathbb{R}^{2}\right)$ with $H \geq H(0)=0$ and it is uniformly convex on the plane (i.e. there exists $a>0$ such that $H_{p p} \geq a I$ ), then continuous viscosity solutions of (1) over $\Omega \subseteq \mathbb{R}^{2}$ are in $C^{1}(\Omega)$. When $n>2$, viscosity solutions are

Key words and phrases. Aronsson Equation, Viscosity Solutions, $C^{1}$ Regularity Problem, Explicit solutions, Calculus of Variations in $L^{\infty}$. 
linearly approximatable at all scales in the sense of De Pauw-Koeller [DePK], having approximate gradients. In the special case of $\Delta_{\infty}$ and when $n=2$, solutions are $C^{1+\alpha}$ (Savin [S], Evans-Savin [E-S]). Recently, Evans and Smart established everywhere differentiability of $\infty$-Harmonic functions [E-Sm].

Herein we prove that when a level set $\{H=c\}$ of $H$ contains a straight line segment, there exists an entire viscosity solution of (1) given as superposition of a linear term plus a rather arbitrary Lipschitz continuous term. The latter may not be $C^{1}$; moreover, it may well be nowhere twice differentiable with Hessian realized only as singular distribution and not as Radon measure, as we show by examples.

We note that our only assumption is $H$ being constant along a line segment but arbitrary otherwise. This suffices for these solutions to appear. Actually, they arise as almost everywhere solutions of the Hamilton-Jacobi equation

$$
H(D u)=c .
$$

In order to keep the proof self-contained and direct, we work with the second order PDE (1) ignoring the relation between viscosity solutions of (1) and differentiable solutions of (3). We just notice that in the classical $C^{2}$ context, the identity

$$
D^{2} u: H_{p}(D u) \otimes H_{p}(D u)=H_{p}(D u) \cdot D(H(D u))
$$

suffices to imply $\mathcal{A}[u]=0$, whenever $H(D u)=c$. Let us now state our result.

Theorem 1. We assume that $H \in C^{1}\left(\mathbb{R}^{n}\right), n \geq 2$ and there exists a straight line segment $[a, b] \subseteq \mathbb{R}^{n}$ along which $H$ is constant. Then, for any $f \in W_{\text {loc }}^{1, \infty}(\mathbb{R})$ satisfying $\left\|f^{\prime}\right\|_{L^{\infty}(\mathbb{R})}<1$, the formula

$$
u(x):=\frac{b+a}{2} \cdot x+f\left(\frac{b-a}{2} \cdot x\right), \quad x \in \mathbb{R}^{n},
$$

defines an entire viscosity solution $u \in W_{l o c}^{1, \infty}\left(\mathbb{R}^{n}\right)$ of the Aronsson equation.

We deduce that the existence of the non- $C^{1}$ solutions (5) implies the following

Corollary 2. Strict level convexity of the Hamiltonian $H$ is necessary to obtain $C^{1}$ regularity of viscosity solutions to the Aronsson PDE in all dimensions $n \geq 2$.

In particular, the uniform convexity assumption of Wang and $\mathrm{Yu}$ [W-Y] can not be relaxed to mere convexity, unless if strict level-convexity is additionally assumed.

We observe that $C^{1}$ regularity of solutions is not an issue of regularity of $H$; the singular solutions (5) persist even when $H \in C^{\infty}\left(\mathbb{R}^{n}\right)$. The sensitive dependence of regularity on the convexity of $H$ is a result of the geometric degeneracy structure of the PDE $\mathcal{A}[u]=0$ which in view of (4) can be rewritten as the perpendicularity condition $H_{p}(D u) \perp D(H(D u))$. Also, the singular solutions persist for arbitrarily small straight line segments, as long as the segments do not trivialize to a point.

\section{Proofs}

For the definition and the properties of viscosity solutions we refer to CrandallIshii-Lions [CIL]. We will first prove Theorem (1) for smooth functions $f$ and then deduce the full result by approximation.

Lemma 3. Let $u$ be given by (5) with $f \in C^{2}(\mathbb{R})$ satisfying $\left\|f^{\prime}\right\|_{L^{\infty}(\mathbb{R})}<1$. Then, (i) $D u\left(\mathbb{R}^{n}\right) \subseteq(a, b)$, i.e. the range of its gradient $D u$ is valued in the open segment $(a, b)=\left\{x \in \mathbb{R}^{n} \mid x=\lambda a+(1-\lambda) b, \lambda \in(0,1)\right\}$, 
(ii) $H_{p}\left(D u\left(\mathbb{R}^{n}\right)\right) \subseteq(\operatorname{span}[b-a])^{\perp}$, i.e. the gradient of $H$ restricted on $D u\left(\mathbb{R}^{n}\right)$ is normal to $(a, b)$.

Proof of Lemma 3. By differentiating (5), we have

$$
D u(x)=\frac{b+a}{2}+\frac{1}{2} f^{\prime}\left(\frac{b-a}{2} \cdot x\right)(b-a),
$$

for all $x \in \mathbb{R}^{n}$. By rearranging (6), we have

$$
D u(x)=\left(\frac{1}{2}-\frac{1}{2} f^{\prime}\left(\frac{b-a}{2} \cdot x\right)\right) a+\left[1-\left(\frac{1}{2}-\frac{1}{2} f^{\prime}\left(\frac{b-a}{2} \cdot x\right)\right)\right] b .
$$

Since $\left\|f^{\prime}\right\|_{L^{\infty}(\mathbb{R})}<1$, there exists a $\delta>0$ such that

$$
\delta \leq \frac{1}{2}-\frac{1}{2} f^{\prime}\left(\frac{b-a}{2} \cdot x\right) \leq 1-\delta,
$$

for all $x \in \mathbb{R}^{n}$. Hence, $D u(x)$ is for all $x \in \mathbb{R}^{n}$ a strict convex combination of $a$ and $b$. Thus, (i) follows. Since $H$ is constant on $[a, b]$, there exists $c \in \mathbb{R}$ such that, for all $t \in(0,1)$, we have

$$
H(t b+(1-t) a)=c .
$$

Since $H \in C^{1}\left(\mathbb{R}^{n}\right)$, we may differentiate to find

$$
\frac{d}{d t}(H(t b+(1-t) a))=(b-a) \cdot H_{p}(t b+(1-t) a)
$$

for all for $0<t<1$. Hence, we obtain that $(b-a) \cdot H_{p}(q)=0$ for all $q \in(a, b)$. Since by (i) we have $D u\left(\mathbb{R}^{n}\right) \subseteq(a, b)$, (ii) follows as well.

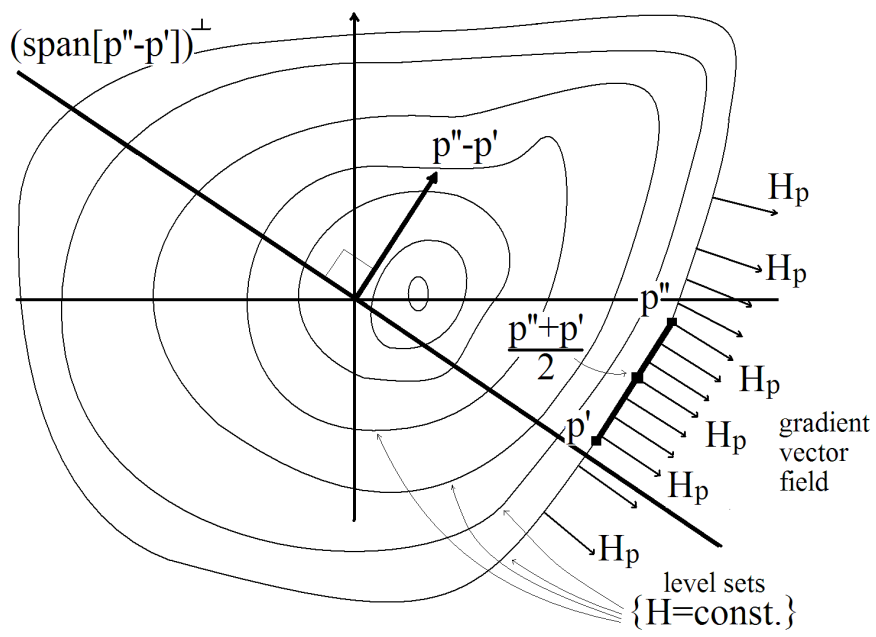

Lemma 4. Let $u$ be given by (5) with $f \in C^{2}(\mathbb{R})$ satisfying $\left\|f^{\prime}\right\|_{L^{\infty}(\mathbb{R})}<1$. Then, (5) defines a $C^{2}\left(\mathbb{R}^{n}\right)$ solution of the Aronsson PDE (1).

Proof of Lemma 4. By (5) and our assumption, the Hessian $D^{2} u(x)$ exists for all $x \in \mathbb{R}^{n}$. By differentiating (6), we have

$$
D^{2} u(x)=\frac{1}{4} f^{\prime \prime}\left(\frac{b-a}{2} \cdot x\right)(b-a) \otimes(b-a) .
$$


We now calculate using (11) and (6):

$$
\begin{aligned}
\mathcal{A}[u](x)= & D^{2} u(x): H_{p}(D u(x)) \otimes H_{p}(D u(x)) \\
= & \frac{1}{4} f^{\prime \prime}\left(\frac{b-a}{2} \cdot x\right)(b-a) \otimes(b-a): \\
& : H_{p}\left(\frac{b+a}{2}+\frac{1}{2} f^{\prime}\left(\frac{b-a}{2} \cdot x\right)(b-a)\right) \otimes \\
& \otimes H_{p}\left(\frac{b+a}{2}+\frac{1}{2} f^{\prime}\left(\frac{b-a}{2} \cdot x\right)(b-a)\right) .
\end{aligned}
$$

By employing Lemma 3, we have

$$
\begin{aligned}
\mathcal{A}[u](x)= & \left\{(b-a) \cdot H_{p}\left(\frac{b+a}{2}+\frac{1}{2} f^{\prime}\left(\frac{b-a}{2} \cdot x\right)(b-a)\right)\right\}^{2} . \\
& \cdot \frac{1}{4} f^{\prime \prime}\left(\frac{b-a}{2} \cdot x\right) \\
= & 0
\end{aligned}
$$

and the Lemma follows.

Hence, in the case of smooth $u$ the PDE (1) is satisfied because the Hessian $D^{2} u$ is normal to $H_{p}(D u) \otimes H_{p}(D u)$ in the space of symmetric matrices. Now we conclude with the general case of merely Lipschitz $f$.

Proof of Theorem 1. Let $u$ be given by (5) with $f \in W_{l o c}^{1, \infty}(\mathbb{R})$ and $\left\|f^{\prime}\right\|_{L^{\infty}(\mathbb{R})}<1$. Let $\eta^{\varepsilon}, \varepsilon>0$, be the standard mollifier and define $f^{\varepsilon}:=f * \eta^{\varepsilon} \in C^{\infty}(\mathbb{R})$. Let also $u^{\varepsilon}$ be given by (5) with $f^{\varepsilon}$ in the place of $f$. Then, $f^{\varepsilon} \longrightarrow f$ in $C^{0}(\mathbb{R})$ as $\varepsilon \rightarrow 0$ and hence $u^{\varepsilon} \longrightarrow u$ in $C^{0}\left(\mathbb{R}^{n}\right)$ as $\varepsilon \rightarrow 0$. Moreover,

$$
\left\|f^{\varepsilon^{\prime}}\right\|_{L^{\infty}(\mathbb{R})} \leq \underset{x \in \mathbb{R}}{\operatorname{ess} \sup _{\mathbb{R}}} \int_{\mathbb{R}}\left|f^{\prime}(x-y)\right|\left|\eta^{\varepsilon}(y)\right| d y
$$

and hence $\left\|f^{\varepsilon^{\prime}}\right\|_{L^{\infty}(\mathbb{R})} \leq\left\|f^{\prime}\right\|_{L^{\infty}(\mathbb{R})}<1$. Consequently, by Lemmas 3 and 4 , all $u^{\varepsilon}$ are smooth entire solutions to PDE $(1): \mathcal{A}\left[u^{\varepsilon}\right]=0$. By the stability of viscosity solutions, we have $\mathcal{A}[u]=0$ on $\mathbb{R}^{n}$ in the viscosity sense and Theorem 1 follows.

Example 5. The choice $f(t):=\frac{1}{2}|t|$ for $|t| \leq 1$ and $f(t+2)=f(t)$ gives a non- $C^{1}$ solution $u$ to the PDE (1). The choice $f(t):=\frac{1}{2} \int_{0}^{t} K_{\alpha, \nu}(s) d s$ with $K_{\alpha, \nu} \in C^{0}(\mathbb{R})$ the singular function of $[\mathrm{K}]$ gives a nowhere twice differentiable solution $u$ to (1) with $D^{2} u$ existing only as a singular first order distribution.

Acknowledgement. Part of this work was carried out when the Author was a doctoral student at the Department of Mathematics, University of Athens, Greece.

\section{REFERENCES}

Ar1. G. Aronsson, On Certain Singular Solutions of the Partial Differential Equation $\left(u_{x}\right)^{2} u_{x x}+$ $2 u_{x} u_{y} u_{x y}+\left(u_{y}\right)^{2} u_{y y}=0$, Manuscripta Math. 47 (1984), no 1-3, $133-151$.

Ar2. G. Aronsson, Construction of Singular Solutions to the p-Harmonic Equation and its Limit Equation for $p=\infty$, Manuscripta Math. 56 (1986), 135 - 158.

BEJ. E. N. Barron, L. C. Evans, R. Jensen, The Infinity Laplacian, Aronsson's Equation and their Generalizations, Transactions of the AMS, Vol. 360, Nr 1, Jan 2008, electr/ly published on July 25, 2007.

CIL. M. G. Crandall, H. Ishii, P.-L. Lions, User's Guide to Viscosity Solutions of 2nd Order Partial Differential Equations, Bulletin of the AMS, Vol. 27, Nr 1, Pages 1 - 67, 1992. 
DePK. T. De Pauw, A. Koeller, Linearly Approximatable Functions, Proc. of the AMS, April 2009, 1347 - 1356, electr. published on Oct. 6, 2008.

E-S. L. C. Evans, O. Savin, $C^{1, \alpha}$ Regularity for Infinity Harmonic Functions in Two Dimensions, Calc. Var. 32, 325 - 347, (2008).

E-Sm. L. C. Evans, C. K. Smart, Everywhere differentiability of Infinity Harmonic Functions, preprint.

GWY. R. Gariepy, Ch. Wang, Y. Yu, Generalized Cone Comparison Principle for Viscosity Solutions of the Aronsson Equation and Absolute Minimizers, Communications in PDE, 31, 1027 - 1046, 2006.

K. N. I. Katzourakis, A Hölder Continuous Nowhere Improvable Function with Derivative Singular Distribution, preprint.

S. O. Savin, $C^{1}$ Regularity for Infinity Harmonic Functions in Two Dimensions, Arch. Rational Mech. Anal. 176, 351 - 361, (2005).

W-Y. C. Wang, Y. Yu $C^{1}$ Regularity of the Aronsson Equation in $\mathbb{R}^{2}$, Ann. Inst. H. Poincaré, AN 25, $659-678,(2008)$.

BCAm - Basque Center for Applied Mathematics, Biskaia Technology Park, Building 500, E-48160, Derio, Spain

E-mail address: nkatzourakis@bcamath.org 